\title{
How to analyze the diagnostic value of sentinel node biopsy in head and neck cancer
}

\author{
Remco de Bree
}

Accepted: 10 December 2012/ Published online: 16 January 2013

(C) Springer-Verlag Berlin Heidelberg 2013

In an attempt to more reliably select the lymph nodes that potentially contain metastases, the sentinel lymph node (SLN) concept has been introduced. This has been studied extensively in melanoma and breast cancer, but was recently introduced in the staging of oral squamous cell carcinoma (OSCC). The SLN is likely to be the first lymph node to harbour metastasis and can be used to provide information on the rest of the nodal basin. The SLN is usually identified by peritumoural injection of radioactive colloid and a blue dye. Preoperative lymphoscintigraphy, intraoperative visualization of blue coloration and intraoperative radionuclide detection using a gamma probe allow identification of the SLN. After surgical removal, this SLN is investigated by meticulous histopathological examination using stepped serial sectioning and immunohistochemistry. If the SLN contains metastatic tumour cells, treatment of the neck is recommended, usually in a second procedure [1]. The SLN procedure is considered to be more precise than imaging procedures and less invasive than elective neck dissection. Moreover, it is associated with significantly less postoperative morbidity and better shoulder function as compared with elective neck dissection [2]. Current best practice guidelines for the provision of SLN biopsy (SLNB) in early OSCC patients have been outlined, which provide a framework for the currently evolving recommendations for its use [3].

The sentinel node concept in OSCC has been validated in several studies in which in all patients after SLNB, a

\footnotetext{
R. de Bree $(\bowtie)$

Department of Otolaryngology/Head and Neck Surgery,

VU University Medical Center, De Boelelaan 1117,

1081 HV Amsterdam, PO Box 7057, 1007 MB Amsterdam,

The Netherlands

e-mail: r.bree@vumc.nl
}

neck dissection was performed. The histopathological examination of the neck dissection specimen was used as reference (gold) standard. Although several European studies had validated the sentinel node concept in OSCC, an American multicenter validation study was initiated in which routine histopathological examination of the neck dissection specimen was used as gold standard. This American College of Surgeons Oncology Group (ACOSOG) Z0360 validation study with 140 patients in 25 institutions showed a sensitivity of $90 \%$ and a negative predictive value (NPV) of $96 \%$ [4]. Because routine histopathological examination (and not step-serial sectioning and immunohistochemistry) of the neck dissection specimen was used as the gold standard occult micrometastases might have been missed [5], potentially contributing to higher figures for sensitivity and negative predicting value.

After initial studies to validate the SLN concept in early OSCC patients, several small prospective observational studies have been reported. In these studies, a neck dissection was performed only when the SLN contained a metastasis and a watchful waiting strategy was followed when the SLN was tumour-free. Two larger single centre studies found sensitivities and NPVs above $90 \%[6,7]$.

In an European multicenter study [8] of $134 \mathrm{cT} 1 / 2 \mathrm{~N} 0$ OSCC patients 79 patients underwent SLNB as the sole staging tool, while 55 patients underwent SLNB followed by elective neck dissection (END). For the two groups together, using a reference standard of 5 years follow-up after SLNB staging, a sensitivity of $91 \%$ and a NPV of $95 \%$ were found. The better performance of the SLNB-assisted END group (sensitivity $96 \%$, NPV $97 \%$ ) compared to the SLNB-alone group (sensitivity $87 \%$, NPV $94 \%$ ) can be explained by the use of standard (routine) histopathological examination of the neck dissection specimen versus 5 years follow-up as a gold standard for metastasis [8]. 
In 2005, Paleri et al. [9] reported on a meta-analysis of 19 pilot validation studies (histopathological examination of neck dissection specimen as gold standard) with a total of 347 oral and oropharyngeal cancer patients and found pooled sensitivity of $92.6 \%$. In this issue of the European Archives of Otorhinolaryngology ... et al. [10] reported a pooled sensitivity of $95 \%$ and a NPV of $96 \%$ in a metaanalysis of 25 validation studies (SLNB followed by concurrent neck dissection as gold standard) with totally 738 head and neck cancer patients. In their meta-analysis studies in which no concurrent END was performed after a negative SLNB were not included, but summarized in Table 3 [10]. The pooled sensitivity and NPV of these studies in which observation was the gold standard were 86 and $94 \%$, respectively, which is slightly lower than the studies using histological examination of the neck dissection specimen as gold standard. This suggests that observation is probably a better gold standard, because micrometastasis and single tumour cells which may be missed during (routine) histopathological examination of the neck dissection specimen and would, if left untreated, proliferate gradually and be detected sooner or later as overt metastases [5].

In both meta-analyses and the ACOSOG Z0360 study, the number of patients per center is below 30. Civantos et al. [4] reported better results of experienced surgeons in the ACOSOG Z0360 study. Ross et al. [11] reported on the first international conference on SLNB in head and neck cancer that centers who had performed 10 or less cases had a lower sensitivity $(57 \%)$ in comparison with centres that had performed more than 10 cases (sensitivity $94 \%$ ). In small series the contribution of the first 10 patients on the results may be substantial. If only larger studies would have been included in the meta-analyses the pooled sensitivity and NPV may have even been higher.

Three large multicenter prospective studies with the SLN procedure as staging technique of the neck in early oral cancer [Sentinel Node European Trial (SENT), the Danish national group trial (DAHANCA 22) and the Brazilian head and neck group] have been started. The observational multicenter SENT study with more than 300 patients, has completed accrual and is waiting for longterm follow-up [12]. In this study, centres were only allowed to include patients after a learning curve of ten sentinel node procedures with concurrent neck dissection.

Histopathological examination is often considered to be the best gold standard. However, histopathological examination may miss minimal disease especially if routine techniques and not step-serial sectioning and immunohistochemistry are used [5]. Therefore, long-term observation of the untreated neck (no neck dissection or radiotherapy) is the best gold standard for determining the value of diagnostic techniques (e.g. the SLN procedure) for detection of occult lymph node metastases.
Another factor influencing the accuracy of a diagnostic technique is the incidence of the disease. In the detection of occult lymph node metastases, if the incidence of metastasis is low the NPV will be high almost regardless of the performance of the diagnostic technique. An upstage by a positive SLN of $30 \%$ as reported in [10] shows that the studied patient population reflects the daily clinical practice and the results of the study can be implemented in routine clinical practice.

Many factors may influence the report of the SLN procedure. Step-serial sectioning and immunohistochemistry of the SLN can significantly improve the sensitivity and NPV. Histopathological examination of the surgical specimen (especially if no step-serial sectioning and immunohistochemistry are used) in stead of long-term observation without treatment of the neck (if SLN is tumor-free) may results in (unreal) higher sensitivity and NPV. Also a low incidence of occult lymph node metastases is easily accompanied by a higher NPV. Surgeons more experienced in SLNB produce probably better accuracy data. The best study design to analyze the real diagnostic value of SLNB in head and neck cancer includes a large number of patients who underwent SLNB by experienced surgeons in multiple centers, with step-serial sectioning and immunohistochemistry and long-term observation without treatment of the neck if the SLN is negative. Since, the results of this kind of studies are not available yet, meta-analyses as published in this issue of the European Archives of Otorhinolaryngology provides valuable information on the potential diagnostic value of SLNB in patients with early oral carcinoma.

\section{References}

1. Leusink FK, van Es RJ, de Bree R et al (2012) Novel diagnostic modalities for assessment of the clinically node-negative neck in oral squamous-cell carcinoma. Lancet Oncol 13(12):E554-E561. doi:10.1016/S1470-2045(12)70395-9

2. Murer K, Huber GF, Haile SR, Stoeckli SJ (2011) Comparison of morbidity between sentinel node biopsy and elective neck dissection for treatment of the n0 neck in patients with oral squamous cell carcinoma. Head Neck 33:1260-1264

3. Alkureishi LW, Burak Z, Alvarez JA et al (2009) Joint practice guidelines for radionuclide. Lymphoscintigraphy for sentinel node localization in oral/oropharyngeal squamous cell carcinoma. Ann Surg Oncol 16:3190-3210

4. Civantos FJ, Zitsch RP, Schuller DE et al (2010) Sentinel lymph node biopsy accurately stages the regional lymph nodes for T1-T2 oral squamous cell carcinomas: results of a prospective multi-institutional trial. J Clin Oncol 28:1395-1400

5. Ferlito A, Shaha AR, Rinaldo A (2002) The incidence of lymph node micrometastases in patients pathologically staged NO in cancer of oral cavity and oropharynx. Oral Oncol 38:3-5

6. Kovacs AF, Stefenelli U, Seitz O et al (2009) Positive sentinel lymph nodes are a negative prognostic factor for survival in T1-2 oral/oropharyngeal cancer-a long-term study on 103 patients. Ann Surg Oncol 16:233-239 
7. Broglie MA, Haile SR, Stoeckli SJ (2011) Long-term experience in sentinel node biopsy for early oral and oropharyngeal squamous cell carcinoma. Ann Surg Oncol 18:2732-2738

8. Alkureishi LW, Ross GL, Shoaib T et al (2010) Sentinel node biopsy in head and neck squamous cell cancer: 5-year follow-up of a European multicenter trial. Ann Surg Oncol 17:2459-2464

9. Paleri V, Rees G, Arullendran P, Shoaib T, Krishman S (2005) Sentinel node biopsy in squamous cell cancer of the oral cavity and oral pharynx: a diagnostic meta-analysis. Head Neck 27:739-747

10. Thompson CF, St John MA, Lawson G, Grogan T, Elashoff D, Mendelsohn AH (2012) Diagnostic value of sentinel lymph node biopsy in head and neck cancer: a metaanalysis. Eur Arch Otorhinolaryngol [Epub ahead of print]

11. Ross GL, Shoaib T, Soutar DS (2002) The First International Conference on Sentinel Node Biopsy in Mucosal Head and Neck Cancer and adoption of a multicenter trial protocol. Ann Surg Oncol 9:406-410

12. Gurney BA, Schilling C, Putcha V et al (2012) Implications of a positive sentinel node in oral squamous cell carcinoma. Head Neck 34:1580-1585 